\title{
Kidney Cancer: A New Forum for Impactful Scientific Interactions
}

Kidney cancer is a highly heterogeneous malignancy, whether viewed across populations, within the same individual, or even within the same tumor. This marked heterogeneity in the biological and clinical behavior of renal cell carcinoma (RCC) has confounded many diagnostic and therapeutic strategies. Indeed, it is a biologically fascinating tumor, characterized by an array of different histologic phenotypes each with unique molecular characteristics and clinical outcomes. Although an increasing number of tumors (mainly small renal masses) are diagnosed using minimally invasive interventions, many patients still face life-altering surgical and medical interventions, with some having to confront the hazards of incurable metastatic disease.

Despite the challenges of this relatively uncommon malignancy - which does not even command top billing as one of the top five causes of cancerrelated death globally - there have been remarkable advances in the understanding of its biologic underpinnings and in the way it is diagnosed and managed throughout the disease continuum. Just in the last two decades, kidney cancer has served as a paradigm for multidisciplinary care, rapid drug development, and evidence-based medicine. For instance, in no other malignant solid tumor has there been so many new systemic agents commercially licensed in the past ten years.

It is in this unique context that we launch Kidney Cancer, a new biomedical journal published by IOS Press. Kidney Cancer is focused on novel or emerging high-impact advances in kidney cancer research and management. This journal's broad aim is "to facilitate progress in understanding the epidemiology/etiology, genetics, molecular correlates, pathogenesis, pharmacology, ethics, patient advocacy and survivorship, diagnosis and treatment of tumors of the kidney." One critical goal is to provide a rigorously peer-reviewed venue for the presentation and discussion of research reports, reviews, short communications, and letters-to-theeditor, among others. In addition the journal will feature a number of special features such as timely social media coverage of kidney cancer related news and events, and "clinical trials corner", which will provide a synopsis of new and interesting developments in that arena. To this end, we have organized an exceptional line-up of editorial board members, consisting of the world leaders in the field of kidney cancer research and treatment. Our team is fully committed to ensuring that this journal only publishes high quality and impactful research that spans the spectrum from bench to bedside to public policy, and everything in between. We anticipate that Kidney Cancer will become an invaluable and indispensable platform for the many collaborative, transdisciplinary, and translational efforts to help treat and finally cure this malignancy.

Primo N. Lara, Jr., MD

University of California Davis School of Medicine UC Davis Comprehensive Cancer Center Sacramento, CA, USA

Peter F.A. Mulders, MD, PhD Radboud University Medical Centre Nijmegen, The Netherlands 